\title{
Electrophysiological recordings from the neuromuscular junction of $C$. elegans $^{*}$
}

\author{
Janet E. Richmond ${ }^{\S}$, Department of Biological Sciences, University of \\ Illinois at Chicago, Chicago, IL 60607 USA
}

\section{Table of Contents}

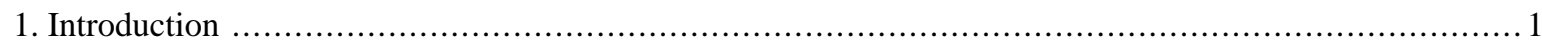

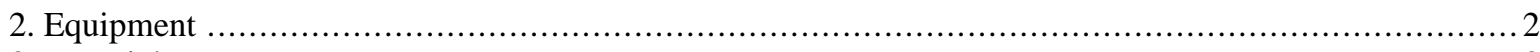

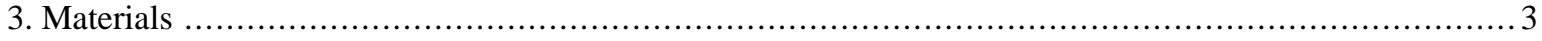

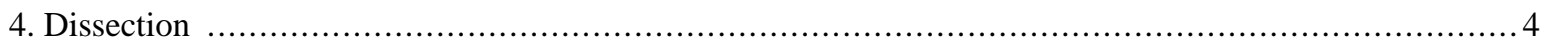

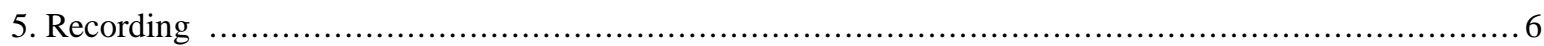

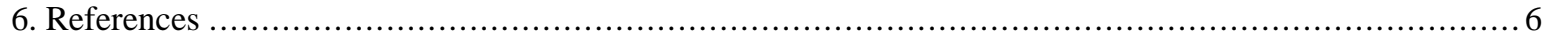

\begin{abstract}
Electrophysiology provides a quantifiable measure of synaptic activity useful in the functional analysis of synaptic proteins. Recent advances in the application of this technique to $C$. elegans provides a means of coupling genetics to electrophysiological analysis, providing new insights into the molecular mechanisms regulating neurotransmission. Here we describe a dissection technique that exposes the neuromuscular junctions of $C$. elegans for electrophysiological analysis. This technique can be adapted to record from virtually any excitable cell in the worm.
\end{abstract}

\section{Introduction}

Neurotransmission is the process by which neurons transfer information via chemical signaling at synaptic contacts with target cells, often on a rapid time scale. Consequently, synaptic transmission requires the coordinated activity of a wide array of proteins. These proteins regulate many aspects of synaptic function including: synapse formation, neuron and muscle excitability, calcium signaling, synaptic vesicle cycling (exocytosis and endocytosis) receptor assembly, localization and function. The electrical events that occur at synapses can be measured using various electrophysiological techniques, providing a quantifiable read out of the activity underlying synaptic activity. By combining this technique with the growing array of molecular and genetic tools available to identify synaptic proteins, we can learn about the molecular machinery responsible for the exquisite precision and fidelity of this process.

\footnotetext{
"Edited by William J. Schafer. WormMethods editor, Victor Ambros. Last revised August 27, 2005. Published October 6, 2006. This chapter should be cited as: Richmond, J.E. Electrophysiological recordings from the neuromuscular junction of C. elegans (October 6, 2006), WormBook, ed. The C. elegans Research Community, WormBook, doi/10.1895/wormbook.1.112.1, http://www.wormbook.org.

Copyright: () 2006 Janet E. Richmond. This is an open-access article distributed under the terms of the Creative Commons Attribution License, which permits unrestricted use, distribution, and reproduction in any medium, provided the original author and source are credited

${ }^{\S}$ To whom correspondence should be addressed. E-mail: jer@uic.edu
} 
C. elegans is a powerful system in which to study the molecular basis of neurotransmission. The challenges of applying electrophysiological techniques to this nematode stem largely from its small size (adults are only $1 \mathrm{~mm}$ in length) and the fact that the worms have high internal hydrostatic pressure and burst upon incision. However, in recent years electrophysiological techniques have been developed that add to the wealth of analytical tools available to study neurotransmission in this organism (reviewed in Francis et al., 2003).

The first successful recordings from C. elegans were achieved by Leon Avery by applying extracellular suction pipettes onto the worm's head, providing a read out of pharyngeal activity (Raizen and Avery, 1994; Avery, et al., 1995). Severing the worm's head to expose the pharyngeal muscles also permitted intracellular recordings to be made (Davis, et al., 1995; Davis, et al., 1999; Lee, et al., 1997; Steger, et al., 2005). Recordings from the pharynx have provided insights into many proteins required for the neuronal control and muscle excitability regulating pharyngeal pumping (Nonet, et al., 1997; Nonet, et al., 1998; Saifee, et al., 1998; Dent, et al., 1997). However, the body wall muscles and neurons of $C$. elegans are much smaller and less accessible than the pharynx, limiting the usefulness of intracellular recordings to the pharynx. Therefore, alternative approaches have been developed that preserve the integrity of these cells, allowing single cell recording in both voltage-clamp and current-clamp. The initial split-open preparation was developed by Goodman and Lockery (Goodman, et al., 1998; Lockery and Goodman, 1998). By applying a cyanoacrylic glue to immobilize the worm, a sharp glass needle could be used to puncture the worms tough cuticle allowing neuronal cell-bodies to be forced up through the incision under hydrostatic pressure. These exposed neurons could then be patch-clamped (Nickell, et al., 2002). This technique has recently been applied to record touch response currents from PLM mechanosensory neurons in the mid-body region (O'Hagan, et al., 2005). The dissection described below to expose the neuromuscular junctions of $C$. elegans developed by Richmond and Jorgensen (Richmond and Jorgensen, 1999) builds on this technique as well as additional insights derived from the Drosophila embryo dissection pioneered by Kendal Broadie (Broadie, 1994). Electrophysiological access to the neuromuscular junction has provided excellent opportunities to study the molecular basis of neurotransmitter release (Richmond and Jorgensen, 1999; Richmond, et al., 1999; Wang, et al., 2001; Koushika, et al., 2001; Weimer, et al., 2003; Lesa, et al., 2003; Schuske, et al., 2003) since release can be stimulated using presynaptic electrodes as well as post-synaptic receptor function (Richmond and Jorgensen, 1999; Culetto, et al., 2004; Gally, et al., 2004; Touroutine, et al., 2005; Towers, et al., 2005; Francis, et al., 2005; Bamber, et al., 2005) and muscle excitability (Jospin, et al., 2002; Kim, et al., 2004; Jospin and Allard, 2004). Variations of the technique below have also been applied to record from head neurons (Brockie, et al., 2001; Mellem, et al., 2002; Zheng, et al., 2004) and vulval muscles (JR unpublished).

\section{Equipment}

A well-equipped, versatile patch-clamp electrophysiology rig comprised of the following equipment is a rather expensive investment $(\sim 75,000)$. Since the worm dissection technique is fairly challenging, it may be advisable to identify an electrophysiology lab in your institution for initial recording attempts prior to purchasing your own rig. This has the added benefit of exposing your lab to electrophysiologists who can provide expertise in the patch clamping technique.

1. Vibration isolation table: For stable recordings it is advisable to purchase an air table which provides an air interface between the room vibrations and the worm preparation. However, a heavy metal plate cushioned by either small tire inner tubes or tennis balls can be substituted and depending on the amount of vibration in your lab may suffice.

2. Farraday Cage: Most labs generate electrical noise, therefore, a Farraday cage that can shield the preparation and the head stage of the amplifier is advisable. These are commercially available but can also be hand-built with a wood frame covered in copper mesh caging.

3. Microscope: We use an Olympus upright microscope (BX51W1) designed for electrophysiologists (the focus knobs are near the front which reduces the chances of knocking the scope and disrupting the patch during refocusing) but this is not essential. The preparation is positioned using a 10X air objective. Recordings are then made using a $40 \mathrm{X}$ water-emersion objective. The working distance of the objective I use is $1.9 \mathrm{~mm}$ which provides enough room to position electrodes. There are longer working distance objectives $(3.8 \mathrm{~mm})$ available but I find that during recordings the meniscus between the objective and the preparation is less likely to break with a $1.9 \mathrm{~mm}$ objective, especially during perfusion, but this depends on the type of recording chamber you 
use. The 40X objective also has DIC (differential interference contrast) providing a clear image of the muscle membrane, which is important in assessing the health of the muscle and during the microelectrode approach onto the membrane. The scope is equipped with a UV light source and GFP filters. C. elegans transgenic animals expressing GFP in either the body wall muscles or nerve cord can be useful indictors of preparation integrity during training.

4. Micromanipulators: Piezo driven manipulators, which allow smooth microelectrode approach and fine control, are recommended (Burleigh or Sutter instruments make great manipulators, we use the Burleigh PCS-5000 series).

5. Amplifier and data acquisition software: There are several commercially available patch clamp amplifiers. We use the HEKA EPC-10 amplifier with Pulse/Pulsefit data acquisition software (now also Patch master) available from HEKA distributors. The leading competitor is Axon Instruments who make patch clamp amplifiers using PClamp/clampex software. Both are good, Axon Instruments has the advantage of providing an excellent mini analysis option, lacking in the HEKA package. If you are not familiar with the patch clamp technique you may want to identify a lab willing to assist you and invest in the amplifier and software used by that lab.

6. Computer: HEKA has PC or Mac options for their software. Axon instruments software is only compatible with PCs.

7. Stimulator: There are several commercially available stimulators. We use a 150-STIM 01D.

8. Pressure-ejection device: There are several commercial pressure-ejection systems available. We use the Picosprtizer III (Parker Instruments).

9. Electrode puller. There are a variety of commercially available pullers. We use the Sutter Instruments Model P-2000, which melts the glass with a laser. Conventional pullers use a heating filament that burns out occasionally or can be damaged during electrode glass insertion/removal and therefore requires periodic adjustments to puller settings, a hassle eliminated with use of the laser puller.

10. Electrode fire-polisher: Fire-polishing the electrode tips used for patch-clamping smoothes and cleans the electrode tip, necessary for good seals to be formed. Polishing also allows adjustment of the electrode-resistance to the required size (I typically use 4 megohm resistance pipettes for the body wall muscle patches). We use an older model Narishige fire polisher that is now obsolete.

\section{Materials}

1. The recording chamber: There are several commercial chambers available but they can also be made. We typically build our own out of 1/16-inch thick plexiglass or magnetic sheet with a circular hole in the middle that is large enough to accommodate a $22 \mathrm{~mm}$ diameter circular cover glass. The dimensions of the chamber will depend on the microscope stage on which the chamber will be held. On the reverse side of the plexiglass chamber we attach a $48 \times 60 \mathrm{~mm}$ cover glass sheet, which we adhere to the plexiglass chamber by placing a pellet of low melt Paraplax tissue embedding wax (available from EM suppliers) in each corner of the cover slip sandwiched between the cover slip and the chamber and gently melt on a hot plate at modest temperatures. Embedding wax melts quickly filling in the space between the chamber and the back glass plate (melt with glass plate facing up), so don't walk away until you have taken the finished chamber off the hot plate. Use a razor blade to remove any wax that may have seeped along the edge of the $22 \mathrm{~mm}$ circular cavity.

2. Sylgard coated cover slips: $22 \mathrm{~mm}$ circular glass cover slips are coated with Sylgard 184 (available from Dow Corning). This coat helps the cyanoacrylic glue adhere to the coverslip when gluing the worms down and provides a cushion in which to clear blocked glue pipettes. We typically make a large batch of cover slips, which last indefinitely in covered containers. The Sylgard is made fresh by mixing 10 parts silicone base to 1 part curing agent by weight. A small Sylgard drop is placed on each cover slip and smeared across using a razor blade edge. The coated coverslips are placed in flat covered containers and left in a 65 degree oven to cure overnight. 
3. Pipettes: The same filament-containing borosilicate glass is used to make the glass needles for the dissection and the muscle recording pipettes. The outer diameter (OD) of the microelectrode glass used will depend on the electrode holder you purchase for recording. Either $1 \mathrm{~mm}$ or $1.5 \mathrm{~mm}$ OD glass is typically used. It is advisable to pull a number of pipettes before proceeding to the dissection step as this stage can require several rapid exchanges of pipettes.

4. Glue: The worms are glued using a cyanoacrylic glue designed for liquid suturing. Although there are several suppliers of liquid suture, the glue consistency varies and to date the optimal product tested is Histoacryl Blue manufactured and sold from B. Braun, Melsungen, Germany.

5. Glue applicator: Glue is applied by mouth-pressure. Polyethylene tubing with an inner diameter that matches the outer diameter of the borosilicate glass $(1$ or $1.5 \mathrm{~mm})$ and about 2 feet long is used. The gluing pipette is inserted into one end of the tubing and the other end holds an eppendorf pipette tip that can be easily replaced if clogged.

6. Extraction pipette: The internal organs of the worm are sucked out by mouth-pressure using a second pipette, in which the tip has been broken back to accommodate worm eggs and gut. This pipette is also inserted into polyethylene tubing with an eppendorf tip at the opposing end.

7. Dissection scope: It is important to have high resolution optics during the dissection. We use a Lieca MZ6 genetics scope that has a $4 \mathrm{X}$ range equipped with a $1.5 \mathrm{X}$ objective and $20 \mathrm{X}$ eyepieces (120X total magnification). A black opaque base disc is used rather than the glass disc used for worm picking and a pair of goose-neck fiber optic cables are used to illuminate the worms from above rather than below.

\section{Dissection}

1. In the recording chamber a circular wax pen line is made to which the Sylgard-coated cover slip is pressed firmly. This immobilizes the cover slip and prevents recording solution from seeping under and dislodging the cover slip during dissection and recording. The chamber is then filled with extracellular recording solution and several adult worms are placed in the center using a worm pick. Worms will thrash, however with practice, these worms can be glued without any further procedures such as cooling to prevent movement.

2. A small amount of glue is placed in a PCR cap held in wax or modeling clay. This small drop of glue can last for several days once the gluing technique has been perfected. Glue pipettes that resemble the dimensions of the patch-clamp electrodes (normally around 4 megohms resistance) are used to apply glue. Using the glue applicator, a small amount of glue is sucked up into the tip of the glue pipette visualizing the tip of the pipette under the dissection scope.

3. The glue polymerizes on contact with solution, therefore it is necessary to maintain a small amount of positive-pressure to the glue pipette to prevent solution from entering and plugging the pipette. As soon as the glue pipette enters the solution the glue needs to be constantly exuded onto the Sylgard under mouth-pressure control. If the pipette is initially plugged or becomes plugged it can often be cleared by gently tapping onto the Sylgard-coated glass coverslip. It is advisable to practice gluing before attempting to glue worms. If you reach the point where you can routinely write your name in a fine line of glue on the Sylgard you are ready to precede to gluing the worm cuticle.

To glue the worm, you need to attach a small amount of glue to either the head or tail and rapidly draw this down onto the surface of the Sylgard (Figure 1-1). Try to do this in the middle of the chamber as worms glued too near the edge will be difficult to reach with the recording pipettes. A constant stream of glue can then be added along the edge of the cuticle from this starting point. Any gaps in the glue should be filled in to prevent the worms from ripping off the Sylgard during the cuticle opening. Once proficient with this step, you will want to make sure the glue is applied to the opposing side of the body from the region you want to record from. For example, we typically record from the ventral-medial body wall muscles, therefore glue is applied along the dorsal cuticle. 


\section{1}
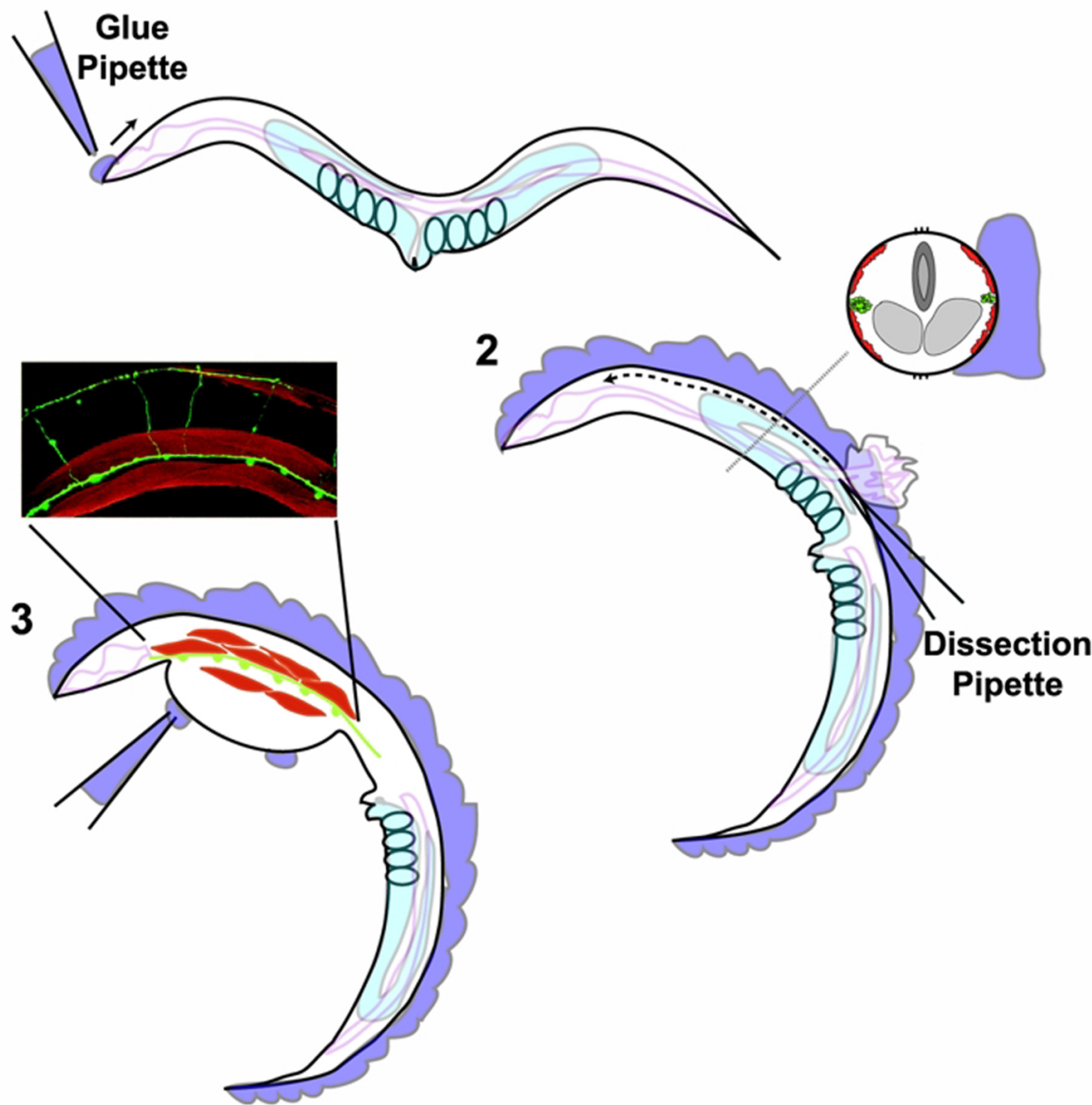

Figure 1. Schematic of the worm dissection. 1. Worms thrashing in extracellular saline within the recording chamber are initially glued to the Sylgard coated cover slip by applying glue to either the head or tail. The glue is then along the cuticle until the entire length of the worm is glued as shown in 2 . The inset shows that the glue forms a stabilizing ridge adjacent to the cuticle. The cuticle incision is made by piercing the cuticle with a sharp glass needle close to the interface with the glue at about mid-body length. The dashed line indicates the progression of the cut towards the head. 3. After sucking out the gonad arm, eggs and intestine from the region exposed by the cut, a clean cuticle flap should be observed. The flap is glued using small spots of glue, exposing the body wall muscles (in red) and ventral nerve cord (green). The inset shows an example of a cut open preparation (GABA neurons labeled with GFP, muscles with Texas red-labeled phaloidin).

4. Once all the worms have been glued, switch to a hand-held dissection pipette. This pipette needs to be sharper than the glue pipette, and have a short sturdy shaft. Using the highest-power on the scope align the pipette parallel to the longitudinal axis of the worm and insert the tip at the mid point of the body, close to the vulva, making the initial incision at the cuticle/glue interface (Figure 1-2). This incision will release the worm's hyprostatic pressure forcing the gut/gonads/eggs out through the incision. Continue cutting the cuticle towards the head of the worm with a slicing motion similar to opening a letter. Once you have made the incision remove all internal organs with the extraction pipette.

5. After clearing the incision, the opened cuticle will retain its cylindrical shape. Using a fresh glue pipette, glue the cut-edge of the cuticle down using minimal glue. The body wall muscles and ventral nerve cord should then be exposed and accessible (Figure 1-3).

6. To remove the basement membrane that covers the muscles, suck off the recording solution and apply collagenase $(0.4 \mathrm{mg} / \mathrm{ml}$ in extracellular recording solution). The Sylgard is hydrophobic and remaining solution will pool around the worms during this process, preventing the worms from drying out. Add a small drop ( 200uL) of collagenase for about 20 seconds, remove and wash several times with recording solution. Proceed rapidly to the recording stage. 


\section{Recording}

1. After positioning the recording chamber on the microscope stage, use the $10 \mathrm{X}$ objective to center the worms. Switch to the $40 \mathrm{X}$ water-emersion objective and find a worm that has intact muscles and nerve cord. Turn the recording chamber so that the longitudinal axis of the worm is parallel with the front of the scope allowing electrodes to be introduced from both the left and right side.

2. Patch the muscle using standard patch-clamping techniques. Electrodes of about 4 megohms resistance work well. Once the electrode contacts the muscle and the pipette resistance increases, apply gentle mouth suction to obtain a gigohm seal. For the whole cell recording configuration, apply more suction and zap the membrane. Body wall muscles of wild-type worms typically have a cell membrane capacitance around $70 \mathrm{pF}$ and remain healthy at a holding potential of $-60 \mathrm{mV}$.

3. The extracellular solution consists of (in $\mathrm{mM}$ ): $\mathrm{NaCl} 150 ; \mathrm{KCl} 5 ; \mathrm{CaCl}_{2} 5 ; \mathrm{MgCl}_{2}$; glucose 10 ; sucrose 5;

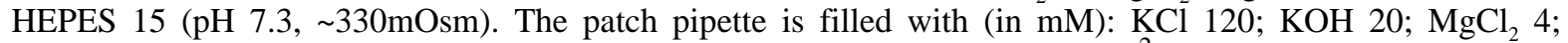
( $N$-tris[Hydroxymethyl] methyl-2-aminoethane-sulfonic acid) $5 ; \mathrm{CaCl}_{2} 0.25 ; \mathrm{Na}^{2} \mathrm{ATP} 4$; sucrose 36; EGTA 5 ( $\mathrm{pH} 7.2, \sim 315 \mathrm{mOsm})$. Note: Using these solutions the muscle chloride gradient is reversed, therefore GABA currents appear as inward currents due to chloride efflux through GABA receptor channels. Variations on these solutions may be desirable to separate the GABA component from the ACh component of the synaptic currents.

\section{References}

Avery, L., Raizen, D., and Lockery, S. (1995). Electrophysiological methods. Methods Cell Biol. 48, $251-269$. Abstract

Bamber, B.A., et al. (2005). The composition of the GABA receptor at the Caenorhabditis elegans neuromuscular junction. Br. J. Pharmacol.

Broadie, K.S. (1994). Synaptogenesis in Drosophila: coupling genetics and electrophysiology. J. Physiol. Paris 88 , 123-139. Abstract Article

Brockie, P.J., et al. (2001). The C. elegans glutamate receptor subunit NMR-1 is required for slow NMDA-activated currents that regulate reversal frequency during locomotion. Neuron 31, 617-630. Abstract Article

Culetto, E., et al. (2004). The Caenorhabditis elegans unc-63 gene encodes a levamisole-sensitive nicotinic acetylcholine receptor alpha subunit. J. Biol. Chem. 279, 42476-42483. Abstract Article

Davis, M.W., et al. (1995). Mutations in the Caenorhabditis elegans Na, K-ATPase alpha-subunit gene, eat-6, disrupt excitable cell function. J. Neurosci. 15, 8408-8418. Abstract

Davis, M.W., et al. (1999). A mutation in the C. elegans EXP-2 potassium channel that alters feeding behavior. Science 286, 2501-2504. Abstract Article

Dent, J.A., Davis, M.W., and Avery, L. (1997). avr-15 encodes a chloride channel subunit that mediates inhibitory glutamatergic neurotransmission and ivermectin sensitivity in Caenorhabditis elegans. EMBO J. 16, 5867-5879. Abstract Article

Francis, M.M., et al. (2005). The Ror receptor tyrosine kinase CAM-1 is required for ACR-16-mediated synaptic transmission at the $C$. elegans neuromuscular junction. Neuron 46, 581-594. Abstract Article

Francis, M.M., Mellem, J.E., and Maricq, A.V. (2003). Bridging the gap between genes and behavior: recent advances in the electrophysiological analysis of neural function in Caenorhabditis elegans. Trends Neurosci. 26, 90-99. Abstract Article

Gally, C., et al. (2004). A transmembrane protein required for acetylcholine receptor clustering in Caenorhabditis elegans. Nature 431, 578-582. Abstract Article

Goodman, M.B., et al. (1998). Active currents regulate sensitivity and dynamic range in C. elegans neurons. Neuron 20,763-772. Abstract Article 
Jospin, M., and Allard, B. (2004). An amiloride-sensitive $\mathrm{H}^{+}$-gated $\mathrm{Na}^{+}$channel in Caenorhabditis elegans body wall muscle cells. J. Physiol. 559, 715-720. Abstract Article

Jospin, M., et al. (2002). The L-type voltage-dependent $\mathrm{Ca}^{2+}$ channel EGL-19 controls body wall muscle function in Caenorhabditis elegans. J. Cell Biol. 159, 337-348. Abstract Article

Kim, H., et al. (2004). SNF-6 is an acetylcholine transporter interacting with the dystrophin complex in Caenorhabditis elegans. Nature 430, 891-896. Abstract Article

Koushika, S.P., et al. (2001). A post-docking role for active zone protein Rim. Nat. Neurosci. 4, 997-1005. Abstract Article

Lee, R.Y., et al. (1997). Mutations in the alpha1 subunit of an L-type voltage-activated $\mathrm{Ca}^{2+}$ channel cause myotonia in Caenorhabditis elegans. EMBO J. 16, 6066-6076. Abstract Article

Lesa, G.M., et al. (2003). Long chain polyunsaturated fatty acids are required for efficient neurotransmission in $C$. elegans. J. Cell Sci. 116, 4965-4975. Abstract Article

Lockery, S.R. and Goodman, M.B. (1998). Tight-seal whole-cell patch clamping of Caenorhabditis elegans neurons. Methods Enzymol. 293, 201-217. Abstract

Mellem, J.E., et al. (2002). Decoding of polymodal sensory stimuli by postsynaptic glutamate receptors in $C$. elegans. Neuron 36, 933-944. Abstract Article

Nickell, W.T., et al. (2002). Single ionic channels of two Caenorhabditis elegans chemosensory neurons in native membrane. J. Membr. Biol. 189, 55-66. Abstract Article

Nonet, M.L., et al. (1997). Caenorhabditis elegans rab-3 mutant synapses exhibit impaired function and are partially depleted of vesicles. J. Neurosci. 17, 8061-8073. Abstract

Nonet, M.L., et al. (1998). Synaptic transmission deficits in Caenorhabditis elegans synaptobrevin mutants. J. Neurosci. 18, 70-80. Abstract

O'Hagan, R., Chalfie, M., and Goodman, M.B. (2005). The MEC-4 DEG/ENaC channel of Caenorhabditis elegans touch receptor neurons transduces mechanical signals. Nat. Neurosci. 8, 43-50. Abstract Article

Raizen, D.M. and Avery, L. (1994). Electrical activity and behavior in the pharynx of Caenorhabditis elegans. Neuron 12, 483-495. Abstract Article

Richmond, J.E., and Jorgensen, E.M. (1999). One GABA and two acetylcholine receptors function at the C. elegans neuromuscular junction. Nat. Neurosci. 2, 791-797. Abstract Article

Richmond, J.E., Davis, W.S., and Jorgensen, E.M. (1999). UNC-13 is required for synaptic vesicle fusion in $C$. elegans. Nat. Neurosci. 2, 959-964. Abstract Article

Saifee, O., Wei, L., and Nonet, M.L. (1998). The Caenorhabditis elegans unc-64 locus encodes a syntaxin that interacts genetically with synaptobrevin. Mol. Biol. Cell 9, 1235-1252. Abstract

Schuske, K.R., et al. (2003). Endophilin is required for synaptic vesicle endocytosis by localizing synaptojanin. Neuron 40, 749-762. Abstract Article

Steger, K.A., et al. (2005). The C. elegans T-type calcium channel CCA-1 boosts neuromuscular transmission. J. Exp. Biol. 208, 2191-2203. Abstract Article

Touroutine, D.V., et al. (2005). acr-16 encodes an essential subunit of the levamisole-resistant nicotinic receptor at the C. elegans neuromuscular junction. J. Biol. Chem. 280, 27013-27021. Abstract Article

Towers, P.R., et al. (2005). The Caenorhabditis elegans lev-8 gene encodes a novel type of nicotinic acetylcholine receptor alpha subunit. J. Neurochem. 93, 1-9. Abstract Article 
Wang, Z.W., et al. (2001). SLO-1 potassium channels control quantal content of neurotransmitter release at the $C$. elegans neuromuscular junction. Neuron 32, 867-881. Abstract Article

Weimer, R.M., et al. (2003). Defects in synaptic vesicle docking in unc-18 mutants. Nat. Neurosci. 6, 1023-1030. Abstract Article

Zheng, Y., et al. (2004). SOL-1 is a CUB-domain protein required for GLR-1 glutamate receptor function in $C$. elegans. Nature 427, 451-457. Abstract Article

All WormBook content, except where otherwise noted, is licensed under a Creative SOMIERIGHISRESERVED Commons Attribution License. 